\title{
Análise do sistema de gerenciamento dos resíduos de serviços de saúde nos municípios da bacia hidrográfica do Rio dos Sinos, Rio Grande do Sul, Brasil
}

\section{Analysis of the management system of the healthcare waste in municipalities of Rio dos Sinos hydrographic basin, Rio Grande do Sul, Brazil}

\author{
Luciana Paulo Gomes \\ Doutora em Engenharia Civil pela Escola de Engenharia de São Carlos da Universidade de São Paulo (USP) - São Carlos (SP), Brasil. Professora Titular do \\ Programa de Pós-graduação em Engenharia Civil da Universidade do Vale do Rio dos Sinos (UNISINOS) - São Leopoldo (RS), Brasil.
}

Roger Vinicius Rosa Esteves

Mestre em Engenharia Civil pela UNISINOS - São Leopoldo (RS), Brasil. Professor da Faculdade Factum - Porto Alegre (RS), Brasil.

\begin{abstract}
Resumo
Nesta pesquisa focou-se o gerenciamento dos resíduos de serviços de saúde (RSS) e especificamente aqueles do tipo perfurocortantes. Foram analisadas as formas atuais de gestão implementadas na bacia hidrográfica do Rio dos Sinos (BHRS), a partir da aplicação de questionários nos estabelecimentos de saúde geradores de RSS. Os resultados indicam que $48,6 \%$ dos estabelecimentos de saúde atendem corretamente à legislação específica brasileira, verificando ainda uma melhor gestão para os estabelecimentos privados. Os estabelecimentos de saúde do tipo "laboratórios, bancos de sangue e farmácias" instalados nos municípios com mais de 20.000 habitantes e área municipal na BHRS dentro da faixa de 80 a 100\% em relação à área total municipal apresentaram os piores resultados em termos de gestão de RSS. O grupo de atividades de serviços de saúde com o maior número de estabelecimentos na BHRS — "consultórios/clínicas de odontologia, clínicas veterinárias, drogarias e unidade móvel" — indicou um dos menores índices de conhecimento das exigências legais específicas relativas ao tema estudado.
\end{abstract}

Palavras-chave: resíduos de serviços de saúde; perfurocortantes; gerenciamento de resíduos.

\section{Abstract}

This research focused on the management of healthcare waste, specifically about the sharps types. The current ways of management in the Rio dos Sinos basin (BHRS) were analyzed with the use of questionnaires in health establishments generators of healthcare waste. The results indicate that $48.6 \%$ of health facilities comply with the specific legislation in Brazil, also indicating a better management coming from private institutions. Health facilities of the type "labs, blood banks and pharmacies" installed in municipalities with over 20,000 inhabitants and municipal area in BHRS within the range of 80 to $100 \%$ relative to the total municipal area had the worst results in terms of healthcare waste management. The group activities of healthcare services with the highest number of establishments in BHRS "dental offices/clinics, veterinary clinics, drugstores and mobile health units" — indicated one of the lowest levels of knowledge about specific legal requirements relating to the theme.

Keywords: healthcare waste; sharps; waste management

\section{Introdução}

Os resíduos de serviços de saúde (RSS), devido às suas características tóxicas e/ou patogênicas, constituem um grande problema para a sociedade e para o meio ambiente. O aumento da preocupação mundial com relação a esse tema tem levado várias nações a reverem suas legislações, tendendo essas a se tornarem cada vez mais restritivas. O cerco tem se fechado principalmente em torno dos geradores, que passam a ser responsabilizados pelos resíduos que produzem, sendo obrigados a destiná-los de forma adequada.

É notória a gama de exigências das normas e legislações brasileiras no que se refere ao gerenciamento dos resíduos dos estabelecimentos de saúde. Porém, na prática, estas nem sempre são cumpridas devido, principalmente, à falta de recursos da instituição, controle e 
fiscalização dos órgãos competentes. Em decorrência disto, grande parte dos estabelecimentos do país não tratam adequadamente seus resíduos, pondo em risco a saúde pública e contaminando o meio ambiente (CAETANO \& GOMES, 2006).

Este estudo reflete a importância de obter maiores informações e propor formas de gerenciamento adequadas dos RSS nos 32 municípios da Bacia Hidrográfica do Rio dos Sinos (BHRS), região densamente povoada, hoje com 1 milhão e 350 mil habitantes. Reconhecidamente a complexidade do gerenciamento desses resíduos e os riscos existentes de contaminação através de agentes físicos, químicos, biológicos, e de acidentes confirmam e justificam os estudos.

\section{Metodologia}

O método empregado nesta pesquisa é o Survey, o qual pode ser descrito como a obtenção de dados ou informações sobre características, ações ou opiniões de determinado grupo de pessoas, indicado como representante de uma população-alvo, por meio de um instrumento de pesquisa, normalmente um questionário (PINSONNEAULT \& KRAEMER, 1992).

Segundo Freitas et al. (2000), esse método é apropriado quando se deseja responder a questões do tipo "o quê?", "por quê?", "como?" e "quando?", ou seja, quando pretende-se entender o que acontece com determinado processo que está sendo avaliado (nesse caso, a partir da aplicação da legislação brasileira sobre RSS nos diferentes estabelecimentos de serviços de saúde na BHRS).

A metodologia utilizada para o desenvolvimento da pesquisa baseou-se em uma análise qualitativa e quantitativa dos dados relativos ao gerenciamento de RSS, nos 32 municípios da BHRS. Foram realizadas visitas às unidades geradoras de RSS, bem como levantamentos técnicos para a busca de informações já publicadas sobre o tema. Além das formas de gerenciamento, para compor a atividade de "gestão" foi realizado o levantamento da legislação brasileira, incluindo a específica do Estado do Rio Grande do Sul, sobre RSS e ainda as normas técnicas brasileiras. Esse levantamento foi realizado junto aos órgãos competentes via busca na internet (sites: www.mma.gov.br; www.anvisa.gov.br; www.fepam.rs.gov.br; www.sema.rs.gov.br) e ABNT.
Consequentemente foi realizado um levantamento de todos os estabelecimentos instalados na BHRS servindo de base para um diagnóstico do sistema, bem como para a determinação do tamanho e tipo de amostra que foi empregada na pesquisa. A busca por estas informações deu-se através dos Conselhos Regionais de Medicina Veterinária, de Farmácia, de Odontologia, Cadastro Nacional de Estabelecimentos de Saúde (CNES) e no Sindicato dos Estabelecimentos Funerários do Rio Grande do Sul. A partir dessas informações foram identificados os estabelecimentos de saúde existentes na BHRS. O resultado indicou 3.254 estabelecimentos, sendo essa a "população" deste estudo.

$\mathrm{Na}$ sequência, os estabelecimentos foram divididos em cinco grupos de atividades geradoras de RSS, a partir das definições indicadas na Resolução CONAMA no 358/05. Hospitais foram classificados como classe A; já na classe B foram agrupados os estabelecimentos do tipo policlínicas, postos de saúde, centros de saúde, pronto atendimento, clínicas de diálise. Na classe C incluíram-se os laboratórios, bancos de sangue e farmácias. Consultório/clínicas de Odontologia, clínicas veterinárias, drogarias e unidade móvel fazem parte da classe D. Para a classe E, foram definidos as funerárias, centro de atenção psicossocial e consultórios médicos. Ainda para cada classe realizou-se a subdivisão dos estabelecimentos na esfera administrativa de gestão: pública ou privada.

Para caracterizar a bacia hidrográfica, os municípios foram agrupados segundo características referentes à população e à área municipal. Como alguns municípios não pertencem na íntegra à BHRS, considerou-se que os mesmos têm implicações na gestão de RSS, diferentes dos demais. Da mesma forma, municípios com maior população geram, consequentemente, mais resíduos e, portanto, influem de forma diferenciada na gestão de RSS. O Quadro 1 apresenta os critérios empregados nesse trabalho para a divisão dos municípios em grupos de prioridades.

Para atender aos objetivos específicos propostos, foi aplicado o instrumento de avaliação do tipo "questionário", seguindo a metodologia Survey. A amostra foi do tipo estratificada e proporcional.

Assim, a "população" empregada nesse trabalho, por classe de estabelecimentos, ficou assim subdividida: 32 estabelecimentos de Classe A; 347 estabelecimentos de Classe B; 305 estabelecimentos

Quadro 1 - Critérios empregados para a divisão dos municípios da bacia hidrográfica do Rio dos Sinos (RS), Brasil, em grupos de prioridades.

\begin{tabular}{|c|c|c|c|c|c|c|}
\hline \multirow{2}{*}{ Municípios } & \multicolumn{3}{|c|}{ População na BHRS (habitantes) } & \multicolumn{3}{|c|}{ \% área municipal na BHRS em relação à área total municipal } \\
\hline & $<20.000$ & 20.000 a 100.000 & $>100.000$ & 0 a 49 & 50 a 79 & 80 a 100 \\
\hline \multirow{2}{*}{ Grupo 1: alto impacto } & & & $x$ & & & $\mathrm{x}$ \\
\hline & & $x$ & & & & $x$ \\
\hline \multirow{4}{*}{ Grupo 2: médio impacto } & & & $x$ & $x$ & & \\
\hline & & & $\mathrm{x}$ & & $\mathrm{x}$ & \\
\hline & & $x$ & & & $x$ & \\
\hline & $x$ & & & & & $x$ \\
\hline \multirow{3}{*}{ Grupo 3: baixo impacto } & $x$ & & & $\mathrm{x}$ & & \\
\hline & & $x$ & & $x$ & & \\
\hline & $\mathrm{x}$ & & & & $\mathrm{X}$ & \\
\hline
\end{tabular}

BHRS: bacia hidrográfica do Rio dos Sinos. 
de Classe C; 2215 estabelecimentos de Classe D, e 355 estabelecimentos de Classe E.

Com esses números, a amostra mínima necessária resultou em 94 estabelecimentos (para erro de 5\%, nível de confiança de 95\% e estimativa de 50\%)

Como são 5 classes de estabelecimentos, acordou-se por amostrar 20 estabelecimentos por classe (A, B, C, D e E), ou seja, a amostra total na BHRS passou a ser 20x5=100 estabelecimentos, sendo 51\% dos estabelecimentos (população total) localizada nos municípios do Grupo 1 (alto impacto), sendo 1 estabelecimento público e 9 privados para cada classe; $20 \%$ dos municípios no Grupo 2 (médio impacto), onde a amostra ficou em 1 estabelecimento público e 3 privados para cada classe de estabelecimento; e 29\% no Grupo 3 de municípios (baixo impacto) sendo 1 estabelecimento público e 5 privados.

\section{Elaboração do questionário}

Foi realizada revisão bibliográfica sobre o tema, de forma a estruturar um primeiro modelo de questionário. Para a escolha das questões, foram consideradas as observações de Wright e Giovinazzo (2000). O questionário foi aprimorado com rodada-teste realizada com cinco profissionais da área da saúde na Unisinos (Farmácia Panvel, Ambulatório, Laboratório de Anatomia, Laboratório de Neurociências e Laboratório de Enfermagem).

Procurou-se, com o questionário, identificar procedimentos adotados nos estabelecimentos quanto ao sistema de gerenciamento adotado para os RSS e conferir o formato das questões envolvidas no instrumento.

O questionário constituiu-se de 28 questões relacionadas à gestão dos estabelecimentos, plano de gerenciamento dos resíduos de serviços de saúde, classificação, segregação, acondicionamento, coleta interna, armazenamento, transporte e, ainda, tratamento e disposição final de RSS. Exceto pela última questão (que buscou avaliar as opções de tratamento dos RSS gerados na BHRS), todas as questões reportam-se para respostas do tipo: "sim" (gestão correta), "não" (gestão inadequada) ou "NA" (não se aplica).

\section{Coleta dos dados}

Os dados da pesquisa foram coletados por meio de entrevistas junto aos estabelecimentos e seus respectivos representantes, sendo o questionário aplicado pelo pesquisador.

\section{Aplicação do questionário}

Em um primeiro momento, tentou-se entrevistar os responsáveis pela gestão dos RSS sendo que apenas 25\% dos estabelecimentos possuíam responsáveis pelos seus resíduos e sabiam o que era um plano de gerenciamento de RSS (PGRSS). Devido à dificuldade de conversar com cada coordenador, os questionários foram aplicados aos funcionários presentes no estabelecimento no momento da entrevista.

\section{Resultados}

O questionário foi aplicado em 97 estabelecimentos de saúde nos municípios da BHRS. A amostra (100 estabelecimentos) não pôde ser completa porque no Grupo 2 (municípios de médio impacto) não foi identificado nenhum estabelecimento público de classificação C (laboratórios, bancos de sangue e farmácias) e, da mesma forma, no Grupo 3 (municípios de pequeno impacto) não existia estabelecimento público algum do tipo A (hospitais) e nem estabelecimento público do tipo C.

A Figura 1 apresenta o grau de entendimento sobre todas as etapas do gerenciamento dos RSS, incluídos todos os municípios da BHRS e todos os grupos de estabelecimentos geradores de RSS

A Figura 1 demonstra resultados similares para as respostas corretas (gestão dos RSS adequada, seguindo as boas práticas de gestão e que atendem à legislação ambiental específica). Nesta primeira análise, geral para toda a bacia, 17,8\% das questões acabaram respondidas pela alternativa NA, indicando desconhecimento dos respondentes em relação à legislação específica vigente como, por exemplo, Decreto Federal no 96.044/88; ANVISA 306/04 e Norma CNEN - NE - 3.03/99. Esse resultado também indica que os estabelecimentos da área da saúde precisam de apoio para treinamentos, capacitações e posteriormente a elaboração de PGRSS que atendam à legislação, focando no controle e na eficiência de medidas que minimizem os equívocos verificados na pesquisa. Também seria importante já conferir os resultados de Silva et al. (2005) os quais indicam que o manejo

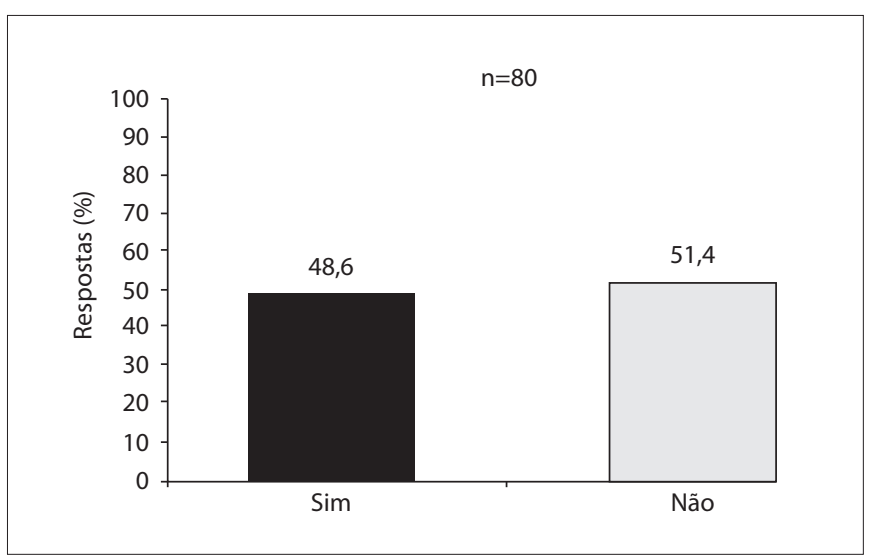

Figura 1 - Resultado de todos os questionários respondidos, representativo do gerenciamento integrado de resíduos de serviços de saúde na bacia hidrográfica do Rio dos Sinos. As questões respondidas com "não se aplica" (17,8\%) foram excluídas do gráfico, de maneira que 100\% das respostas correspondem à soma das alternativas "sim" $(39,9 \%)$ e "não" $(42,2 \%)$. 
inadequado dos resíduos, pode promover a contaminação de toda a massa dos resíduos. Portanto, para que não ocorra a disseminação de doenças é importante que nessa etapa do processo haja um trabalho de conscientização nas unidades internas do estabelecimento relacionado à segregação. Essa conscientização permitirá a redução da quantidade de resíduos infectantes e, consequentemente, das despesas com o tratamento, além de minimizar doenças ocupacionais e taxas de infecção.

\section{Resultados por esfera de gestão administrativa na bacia hidrográfica do Rio dos Sinos}

A Figura 2 divide os estabelecimentos da BHRS por esferas administrativas de gestão: estabelecimentos públicos e privados, indicando o resultado geral do gerenciamento de RSS implantado na BHRS.

De acordo com a Figura 2, pode-se verificar que os estabelecimentos de gestão privada possuem, pelas respostas obtidas, uma gestão mais adequada que a dos públicos. As diferenças entre os resultados dos estabelecimentos públicos e privados, pode passar pela questão "falta de fiscalização" ou "fiscalização mais focada no setor privado" ou ainda porque a forma de contratação de funcionários públicos via concurso (o que traz a estabilidade desses profissionais) pode estar dificultando as mudanças/melhorias de gestão e rotinas operacionais nos estabelecimentos públicos. Diferentemente, no grupo privado, a falta de envolvimento para realizar a implantação das novas regras ambientais é resolvida com a troca dos profissionais.

Segundo Leitão (2006), a falta de políticas ambientais e das resoluções e normas vigentes para o gerenciamento dos resíduos de serviços de saúde traduzida em geral pela ausência de fiscalização

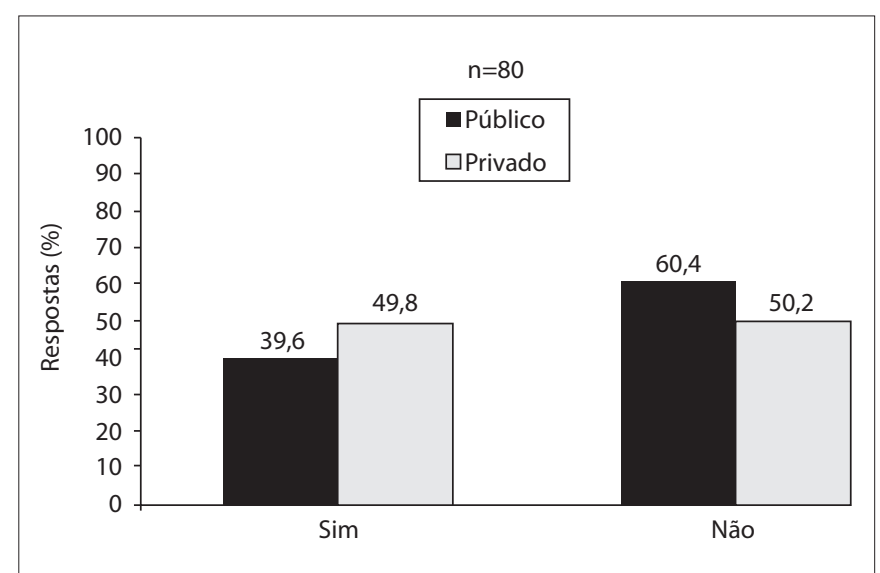

Figura 2 - Resultado de todos os questionários respondidos, representativo do Gerenciamento Integrado de RSS na bacia hidrográfica do Rio dos Sinos por esfera administrativa. As questões respondidas com não se aplica foram excluídas do gráfico, de maneira que $100 \%$ das respostas correspondem à soma das alternativas sim e não. mais eficaz e constante dos órgãos oficiais, não deve impedir a consciência ética dos responsáveis pelos serviços de saúde quanto à elaboração, execução e manutenção de planos que visem reduzir os impactos dos RSS tanto em nível ambiental como ocupacional. O mesmo autor salienta que a gestão inadequada dos RSS é conhecida na maioria das vezes por todas as partes envolvidas faltando portanto um maior envolvimento dos mesmos.

Outro aspecto importante que não deve ser esquecido é a carência de recursos financeiros no sistema público de saúde onde muitas vezes faltam medicamentos, recursos humanos, materiais e até equipamentos para suprir a demanda de atendimento da população. Esse fator pode estar interferindo nas práticas de gestão dos RSS. A falta de vontade política e de entendimento da importância em atender à legislação são fatores que também podem ser citados como causadores da situação precária observada.

Ao compararem-se os três grupos, percebe-se que os estabelecimentos públicos do Grupo 1 obtiveram um resultado melhor (pontuação de 49,2\% de respostas satisfatórias contra 31,5 e 33,3\%, respectivamente para os Grupos 2 e 3). Os resultados parecem indicar que o setor público está melhor instalado nos grandes municípios, havendo um "esquecimento" com os menores. Diferentemente, o setor privado vem atendendo igualmente todos os níveis de municípios, embora ainda com resultados apenas razoáveis (aproximadamente com 50\% de resultados de boa gestão)

As Figuras 3 e 4 apresentam os resultados dos estabelecimentos público e privado, segundo os diferentes temas abordados no questionário.

Os melhores resultados obtidos, nos estabelecimentos públicos da BHRS foram nos temas "segregação" e "transporte". O primeiro tipo é resultante da conscientização e engajamento das pessoas, já o segundo é uma atividade exercida por terceiros, contratados. Assim,

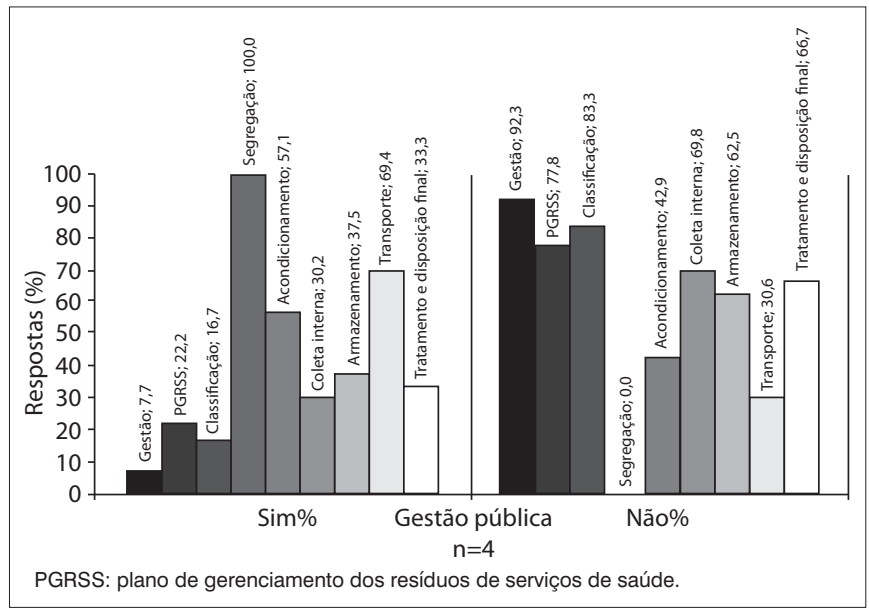

Figura 3 - Resultado de todos os questionários respondidos por etapas da gestão pública. A soma dos valores para as respostas sim e não em um mesmo tema não resulta em $100 \%$ porque a diferença corresponde às questões respondidas com não se aplica. 
pode-se inferir que com a escolha adequada de parceiros e consequente contratação esta etapa do gerenciamento de RSS é razoavelmente bem atendida. Pelos resultados ruins anteriormente apresentados é surpreendente que o tema segregação tenha recebido $100 \%$ de respostas corretas

Pode-se verificar que gestão, PGRSS e classificação são os principais fatores de inconformidades apresentados nos estabelecimentos públicos. São etapas gerenciais que demandam equipes treinadas, conscientizadas e atualizadas na área. A falta de recursos financeiros pode também ser um motivo que justifique esses piores resultados.

Para o setor privado, os melhores resultados foram nas etapas de "segregação" e "acondicionamento", etapas de forte envolvimento com as pessoas que atuam nas atividades geradoras de RSS, demonstrando boa conscientização ambiental.

A "segregação dos RSS" é a etapa que, independentemente da forma de gestão, apresentou os melhores resultados. Este resultado favorece aos funcionários que trabalham no setor de limpeza dos estabelecimentos de serviços de saúde, os quais muitas vezes se acidentam pela má segregação e acondicionamento dos RSS. Segundo Takada (2003), a segurança e o bem-estar das pessoas que manejam os RSS dependem em boa parte, da capacitação e motivação dos médicos, enfermeiros, pacientes e de todo o pessoal que interage na geração dos mesmos. Salomão, Trevizan e Gunther (2004) também reforçaram a importância dessa etapa, já que em seu trabalho verificou que a segregação dos RSS poderia diminuir em $82 \%$ os resíduos infectantes potencializando o aproveitamento dos demais resíduos gerados.

\section{Resultados por tipo de estabelecimentos geradores de resíduos sólidos de saúde nos grupos de municípios na bacia hidrográfica do Rio dos Sinos}

Os resultados globais, por tipo de estabelecimentos geradores de RSS estão indicados na Figura 5. Nela, todas as questões respondidas são apresentadas somadas, indicando como está a gestão de RSS para cada grupo.

Analisando a Figura 5, observa-se que os estabelecimentos do Grupo A obtiveram a maior pontuação com uma média de $77,4 \%$. Isso acontece por serem instituições mais estruturadas, com um número maior de trabalhos de conscientização e fiscalização. Já os

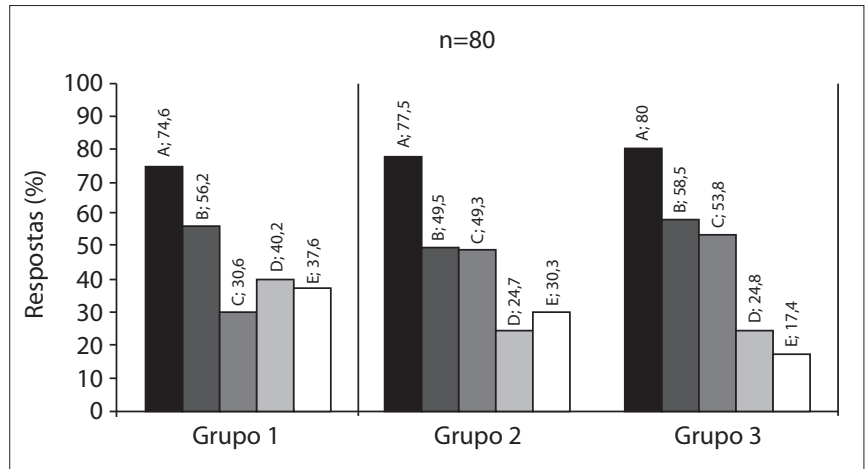

Figura 5 - Resultado de todos os questionários respondidos por estabelecimentos e por grupos de importância na bacia hidrográfica do Rio dos Sinos. As questões respondidas com não se aplica foram excluídas do gráfico, de maneira que 100\% das respostas correspondam a soma das alternativas sim e não.

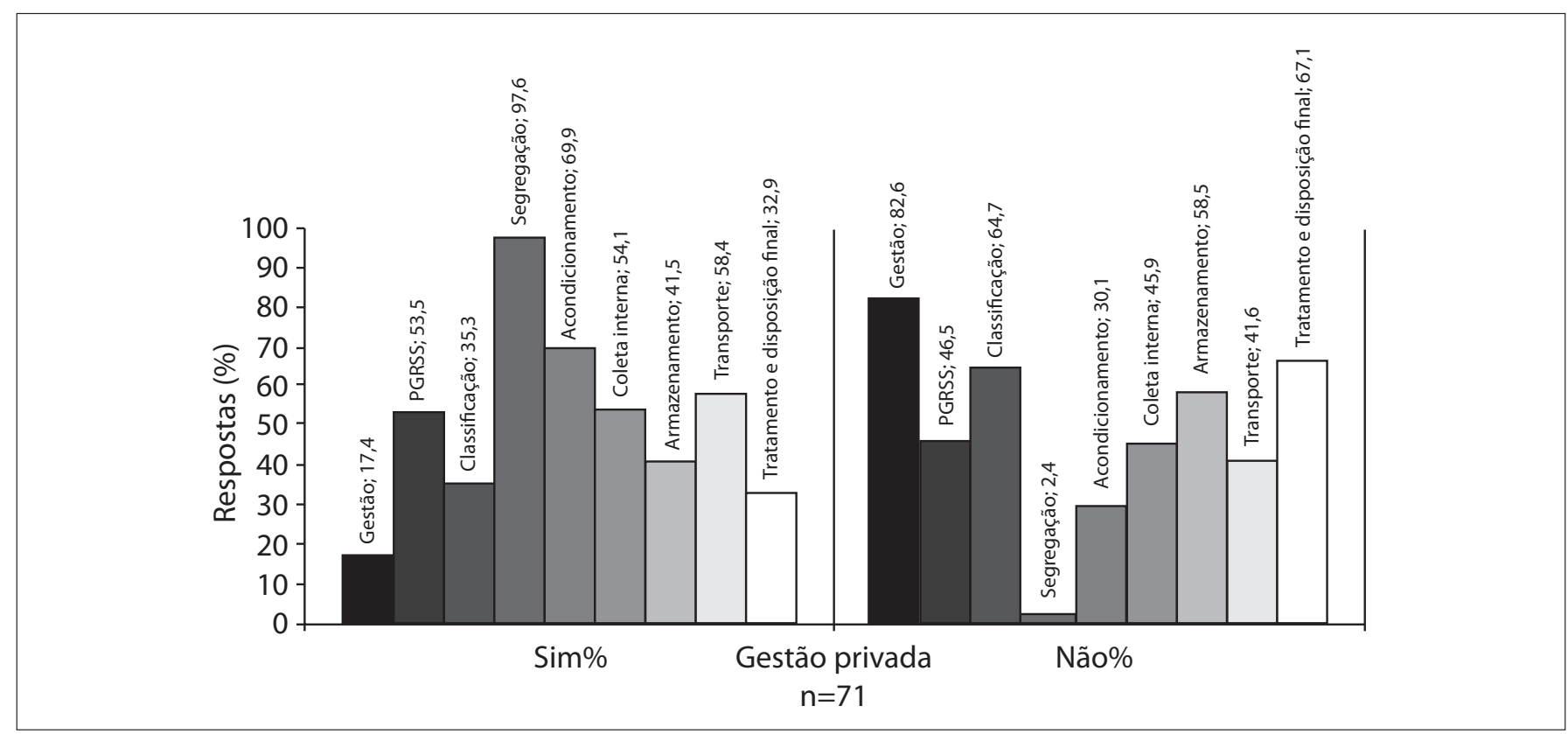

Figura 4 - Resultado de todos os questionários respondidos por etapas da gestão privada. A soma dos valores para as respostas sim e não em um mesmo tema não resulta em 100\% porque a diferença corresponde às questões respondidas com não se aplica. 
estabelecimentos tipo E atingiram a pior média (28,4\%), mostrando a principal categoria que deve ser trabalhada, mesmo que gerando pouca quantidade de resíduo. Cabe ressaltar que os hospitais podem ainda melhorar suas operações de gerenciamento através de uma melhor segregação e utilização de programas de coleta e reciclagem.

Era esperada uma melhor resposta para os estabelecimentos do tipo A, seguido dos tipos B, C, D e E, nesta ordem, supondo-se que o porte e grau de importância nos serviços de saúde deveriam corresponder a uma melhor gestão dos RSS. Do ponto de vista quantitativo, embora não se tenha medido isto, é razoável dizer que a geração decresce com os tipos de estabelecimentos: maior geração nos estabelecimentos tipo A e a menor nos do tipo E. Por outro lado, pelo número de estabelecimentos do tipo D (2.215 estabelecimentos na BHRS) merecem uma melhor atenção especial, seguidos dos tipos E, B e C (355, 347 e 305, respectivamente), contra apenas 32 estabelecimentos do tipo A.

A proposta de ações para melhoria da gestão de RSS na BHRS, com 48,6\% de gerenciamento adequado (Figura 1) passa por uma avaliação de prioridades: será que os investimentos devem focar os estabelecimentos do tipo A, melhorando ainda mais a média da bacia hidrográfica, com esforços localizados e de mais fácil implantação? Ou deve-se sugerir centrar esforços nos estabelecimentos do tipo D com uma das piores avaliações (29,9\%, média de gestão adequada nos 3 grupos de municípios), mas com o maior número de estabelecimentos, espalhados por toda a região? Talvez a resposta não seja nem uma nem outra, mas sim incentivar investimentos e conscientização das equipes e usuários dos estabelecimentos tipos B, C e E, em faixa intermediária em termos de número de estabelecimentos (total de 1.007 na BHRS) e avaliação média de 42,6\%.

\section{Resultados sobre 0 gerenciamento de resíduos sólidos de saúde perfurocortantes}

A Figura 6 resume as respostas indicadas pelos estabelecimentos no que diz respeito ao gerenciamento de perfurocortantes. Foram questionadas as etapas de "classificação", "segregação", "acondicionamento", "coleta interna" e "armazenamento".

Os RSS perfurocortantes são os resíduos que potencialmente podem causar infecções nos setores da saúde, provavelmente devido às formas anatômicas utilizadas, tornando-se um perigo e exigindo atenção no seu manuseio. Analisando a Figura 6, pode-se verificar que, em média, 83,3\% dos estabelecimentos pesquisados acondicionam os resíduos perfurocortantes em recipientes com paredes rígidas, o que confere com as atuais rotinas de segurança e ambientais adequadas. Em alguns estabelecimentos, esses resíduos eram acondicionados em garrafas PET e tonéis plásticos adaptados para esta finalidade. Nos outros estabelecimentos (16,7\%), este resíduo estava acondicionado em sacos plásticos comuns utilizados para os resíduos sólidos urbanos (RSU) sem nenhum tipo de identificação. Essa última situação direciona, provavelmente, para um descarte combinado com RSU, criando situação de risco grave para os funcionários da coleta e gerando impacto ambiental nos locais de destino final de RSU. Essa situação, classificada como de alto potencial de risco confere com os resultados de Silva (2004) que, através de um levantamento realizado pelo Centro de Controle de Doenças e Prevenção em Atlanta (EUA), verificou 57 casos documentados de profissionais de saúde que adquiriram infecção ocupacional por HIV. A maioria desses casos, ou seja, $86 \%$ foram expostos a sangue e $88 \%$ sofreram acidentes percutâneos com perfurocortantes (no estudo existia a possibilidade de

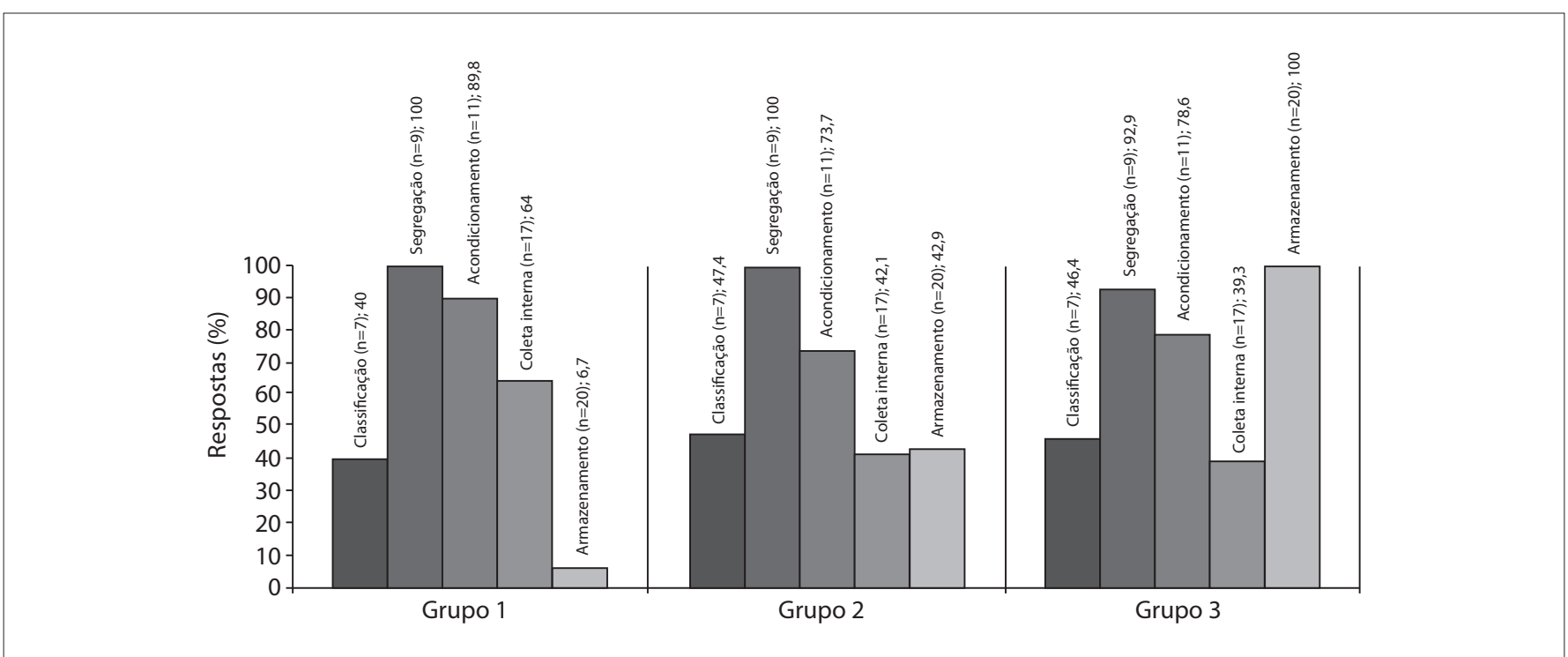

Figura 6 - Resultado de todos os questionários respondidos por grupos de importância na bacia hidrográfica do Rio dos Sinos relacionado a questões de resíduos sólidos de saúde perfurocortantes. As questões respondidas com não se aplica foram excluídas do gráfico, de maneira que $100 \%$ das respostas correspondem à soma das alternativas sim e não. 
responder a mais de um item, por isso a soma dos dados anteriores ser maior que $100 \%$ )

Em relação à segregação dos RSS perfurocortantes, 97,9\% dos estabelecimentos pesquisados segregam-no de maneira adequada. Conforme Silva (2004), a segregação dos resíduos tem como objetivo minimizar a contaminação de resíduos considerados comuns, permitindo a adoção de procedimentos específicos de manejo de cada grupo de resíduos, possibilitando o tratamento específico para cada categoria reduzindo os riscos para a saúde e diminuir os custos para o manejo e tratamento dos mesmos. Nesta mesma linha, Zamoner (2008) e Ferreira et al. (2009) afirmam que a segregação dos materiais perfurocortantes deve ser realizada após a sua utilização, sendo acondicionados em recipientes rígidos identificados e fechados previamente para serem enviados à coleta.

Verifica-se ainda (Figura 6) que os estabelecimentos entrevistados possuem dificuldades na etapa de "armazenamento" por não possuírem abrigos externos para armazenarem de forma adequada os resíduos, previamente à etapa de transporte. Conforme as respostas à questão 20 , os estabelecimentos $(35,7 \%)$ não possuem ambientes individuais para cada tipo de resíduo, conforme preconiza a legislação.

Outra etapa importante a ser destacada é a "coleta interna" dos RSS, onde verificaram-se dificuldade e resistência na utilização de equipamentos de proteção individual, o que pode ocasionar acidentes com a manipulação de perfurantes somada à segregação inadequada dos resíduos. De acordo com Silva (2004), a equipe da limpeza é a mais exposta a esse risco.

\section{Resultados do gerenciamento de resíduos sólidos de saúde: tratamento e disposição final}

Um dos objetivos deste trabalho foi analisar as formas de tratamento e disposição final de RSS empregadas pelos estabelecimentos. A região da BHRS é composta por cinco empresas que prestam o serviço de tratamento e disposição final de RSS. Todas trabalham com tecnologias de tratamento térmico, ou autoclavagem ou incineração, que segundo Eleutério, Hamada e Padim (2008) são as opções de tratamentos mais utilizadas no tratamento dos RSS no Brasil em razão da eficiência de desinfecção e redução de volume.

As informações obtidas durante a pesquisa indicam que os estabelecimentos destinam seus RSS à tecnologia de autoclavagem. A questão "investimento" pode ser a que define o tipo de tratamento. O contrato dos estabelecimentos geradores com as empresas prestadoras desse serviço determina que o valor do serviço seja formado pela quantidade de resíduo gerada e o número de coletas a serem realizadas. Os resíduos tratados por autoclaves possuem um preço médio de R \$2,00 por quilo de resíduo desinfectado. Já os resíduos utilizados no processo de incineração têm como preço médio R 2,50 por quilo podendo ser ajustado em virtude da complexidade operacional.

Em relação ao impacto ambiental, ambas as tecnologias possuem prós e contras. O processo de incineração precisa de monitoramento das emissões gasosas geradas pela queima dos resíduos tratados, de controle na disposição final das cinzas e do lodo gerado pela lavagem dos gases. Já o sistema de autoclavagem tem a desvantagem de resultar em pequena redução do volume de RSS além do processo gerar efluentes líquidos. As empresas prestadoras deste serviço na BHRS possuem trituradores para, após a desinfecção, diminuir esse volume.

Como exemplo de comparação, a Unisinos tem contrato com a Empresa 4 para recolhimento e tratamento (por incineração) de seus resíduos de serviços de saúde. A universidade gera animais mortos contaminados e outros resíduos de ambulatório, além de perfurocortantes (gerados no ambulatório e alguns laboratórios de pesquisa). A geração média mensal é de $100 \mathrm{~kg}$ de RSS, sendo que o investimento é de R\$130,00 por mês. São feitos dois descartes mensais. O valor médio, para descartes eventuais, é de R \$3,10 por kg de RSS, acrescido de R\$ 120,00 como taxa de transporte (dados informados pela empresa para o Sistema de Gestão Ambiental da Unisinos).

Os resultados do questionário indicam a falta da informação do setor sobre o tema, já que apenas 10,2\% dos estabelecimentos informaram a etapa final do gerenciamento dos RSS. Esse resultado confirma os resultados anteriores demonstrando o longo caminho a trilhar na gestão de RSS na BHRS.

\section{Conclusões}

A pesquisa desenvolvida atendeu aos objetivos propostos inicialmente e foi possível concluir que o atendimento legal do setor da saúde gerador de RSS na BHRS, segundo os resultados obtidos nesta pesquisa, é de 48,6\%. Comparando-se as esferas administrativas verificou-se uma melhor gestão para os estabelecimentos de saúde privados em relação aos públicos. Para os municípios de alto impacto (maiores áreas municipais e população), os estabelecimentos de saúde do tipo laboratórios, bancos de sangue e farmácias foram os que apresentaram os piores resultados em termos de gestão de RSS e, para os demais municípios, os consultórios/clínicas de Odontologia, clínicas veterinárias, drogarias e unidades móveis foram os que indicaram o menor conhecimento à cerca das exigências legais específicas relativas ao tema estudado.

Em relação à segregação dos RSS perfurocortantes, 97,9\% dos estabelecimentos pesquisados os segregam de maneira adequada. Apenas 10,2\% dos estabelecimentos informaram conhecer qual seria a etapa final do gerenciamento dos RSS. Dos estabelecimentos respondentes (sobre essa questão exclusivamente), a forma de tratamento de RSS mais utilizada foi a tecnologia de autoclavagem. 


\section{Referências}

BRASIL. (2005) Ministério do Meio Ambiente. Conselho Nacional do Meio Ambiente. Resolução CONAMA n 358, de 29 de abril de 2005. Dispõe sobre o tratamento e a disposição final dos resíduos de serviços de saúde e dá outras providencias. Diário Oficial da União, Brasília, DF, 04 mai. 2005

CAETANO, M.O. \& GOMES, L.P. (2006) Proposta de plano de gerenciamento de resíduos de serviços de saúde para o hospital Beneficência Portuguesa - Porto Alegre - RS. Estudos Tecnológicos, São Leopoldo, v. 2, n. 2, p. 99-112.

ELEUTÉRIO, J.P.L.; HAMADA, J.; PADIM, A.F. (2008) Gerenciamento eficaz no tratamento dos resíduos de serviços de saúde - estudo de duas tecnologias térmicas. XXVIII Encontro Nacional de Engenharia de Produção, 28. 2008, Rio de Janeiro. Resumo. Rio de Janeiro: Enegep.

FERREIRA, M.M.; HISSE, C.N.; SILVEIRA, J.M.A.; SANTANA, B.P. (2009) Resíduos de serviços de saúde: uma abordagem de profissionais da estratégia de saúde da família do RS. Disponível em: <http://www.ufpel. edu.br/cic/2009/cd/pdf/CS/CS_01803.pdf>. Acesso em: 21 jan. 2013.

FREITAS, H.; OLIVEIRA, M.; SACCOL, A.Z.; MOSCAROLA, J. (2000) O método de pesquisa Survey. Revista de Administração, v. 35, n. 3, p. 105-112.

LEITÃO, A.J.C. (2006) Estruturação do plano de gerenciamento de resíduos de senviços de saúde para a farmácia universitária da Universidade Federal Fluminense. Dissertação (Mestrado em Sistemas de Gestão) - Programa de Pós-graduação em Sistemas de Gestão, Universidade Federal Fluminense, Rio de Janeiro.

PINSONNEAULT, A. \& KRAEMER, K.L. (1992) Survey research methodology in management information systems: an assessment. Working Paper \#URB-022. Disponível em: <https://docs.google.com/viewer?a=v\&q= cache:J67dpCP7LgcJ:staf.cs.ui.ac.id/WebKuliah/TKSI/Survey\%2520Me
thod\%2520in\%2520MIS. pdf $+\&$ hl = pt-BR\&gl=br\&pid = bl\&srcid =ADGE ESiaHTxetBeDYTuFKhH9ao5cChc3CmagWTF00BUF677Ol4hkTy88AkS gUxSxD8T7M1bSwwrl55fp0PZX_Gc2skOY44_27L4Qs1yNZZ5yBR9C5 uWGrsDvZt9fazOfWuR_ByWnz9f\&sig=AHIEtbQZsw525W07LAXYxEeitrg bpmfFRA > . Acesso em: 16 jan. 2013.

SALOMÃO, I.S.; TREVIZAN, S.D.P.; GUNTHER, W.M.R. (2004) Segregação de resíduos de serviços de saúde em centros cirúrgicos. Revista Engenharia Sanitária e Ambiental, v. 9, n. 2, p. 108-111.

SILVA, M.F.I. (2004) Resíduos de serviços de saúde - gerenciamento no centro cirúrgico, central de material e centro de recuperação anestésica de um hospital do interior paulista. Tese (Doutorado em Enfermagem) - Programa de Pós-graduação em Enfermagem Interunidades, Escola de Enfermagem de Ribeirão Preto, Universidade de São Paulo, Ribeirão Preto.

SILVA, C.E.; HOPPE, A.E.; RAVANELLO, M.M.; MELLO, N. (2005) Medical wastes management in the south of Brazil. Waste Management, v. 25, p. $600-605$.

TAKADA, A.C.S. (2003) O plano de gerenciamento de resíduos de serviço de saúde e o direito do trabalhador. Monografia (Curso de Especialização em Direito Sanitário para Profissionais de Saúde) Escola Nacional de Saúde Pública Sergio Arouca, Brasília.

WRIGHT, J.T.C. \& GIOVINAZZO, R.A. (2000) Delphi - uma ferramenta de apoio ao planejamento prospectivo. Caderno de Pesquisas em Administração, v. 1, n. 12, p. 54-65.

ZAMONER, M. (2008) Modelo para avaliação de planos de gerenciamento de resíduos de serviços de saúde (PGRSS) para Secretarias Municipais da Saúde e/ou do Meio Ambiente. Ciência \& Saúde Coletiva, v. 13, n. 6, p. 1945-1952. Disponível em: <http://www.scielo.br/pdf/csc/v13n6/ a30v13n6.pdf>. Acesso em: 21 jan. 2013. 